\title{
Sex-Specific Differences in Expression of Histone Demethylases Utx and Uty in Mouse Brain and Neurons
}

\author{
Jun Xu, ${ }^{1,2,3}$ Xinxian Deng, ${ }^{1}$ Rebecca Watkins, ${ }^{3}$ and Christine M. Disteche ${ }^{1}$ \\ ${ }^{1}$ Department of Pathology and Medicine, University of Washington, Seattle, Washington 98195, 2Department of Biomedical Sciences, Cummings School of \\ Veterinary Medicine at Tufts University, North Grafton, Massachusetts 01536, and ${ }^{3}$ Department of Physiological Science, University of California, Los \\ Angeles, California 90095
}

\begin{abstract}
Although X inactivation is thought to balance gene expression between the sexes, some genes escape inactivation, potentially contributing to differences between males and females. Utx (ubiquitously transcribed tetratricopeptide repeat gene on X chromosome) is an escapee gene that encodes a demethylase specific for lysine 27 of histone H3, a mark of repressed chromatin. We found Utx to be expressed higher in females than in males in developing and adult brains and in adult liver. XX mice had a higher level of Utx than XY mice, regardless of whether they had testes or ovaries, indicating that the sexually dimorphic gene expression was a consequence of the sex chromosome complement. Females had significantly higher levels of Utx than males in most brain regions except in the amygdala. The regional expression of the Y-linked paralogue Uty (ubiquitously transcribed tetratricopeptide repeat gene on Y chromosome) was somewhat distinct from that of Utx, specifically in the paraventricular nucleus of the hypothalamus (high Uty) and the amygdala (high $U t x$ ), implying that the two paralogues may be differentially regulated. Higher expression of Utx compared with Uty was detected in P19 pluripotent embryonic carcinoma cells as well as in P19-derived neurons. This transcriptional divergence between the two paralogues was associated with high levels of histone $\mathrm{H} 3$ lysine 4 dimethylation at the Utx promoter and of histone $\mathrm{H} 4$ lysine 16 acetylation throughout the gene body, which suggests that epigenetic mechanisms control differential expression of paralogous genes.
\end{abstract}

Key words: neuron; cognition; Turner syndrome; Klinefelter's syndrome; estrogen; androgen

\section{Introduction}

Males and females differ in physiology and behavior, as well as in brain structure. Such differences are mainly attributed to the action of steroid hormones (Becker et al., 2005). However, not all sexual dimorphisms can be explained as the result of gonadal steroid action (Arnold et al., 2004); nongonadal sources of sexspecific signals include genes on the $\mathrm{X}$ and $\mathrm{Y}$ chromosomes.

$\mathrm{X}$-linked genes have been thought previously to be similarly expressed between the sexes, because $\mathrm{X}$ inactivation silences one of the two X chromosomes in females (Lyon, 1961). However, in humans, at least $15 \%$ of $\mathrm{X}$-linked genes escape inactivation (Carrel and Willard, 2005), and presumably are expressed more highly in females. Some of the $\mathrm{X}$ escapees have paralogues on the Y chromosome (Xu and Disteche, 2006). For the Y paralogue to compensate for the female's second

Received Dec. 5, 2007; revised March 18, 2008; accepted March 18, 2008.

This work was supported by National Institutes of Health (NIH) Grants HD43942 (J.X.) and GM46883 (C.M.D.) as well as a seed grant from the Tufts University Cummings School of Veterinary Medicine (J.X.). We thank Dr. Arthur P. Arnold [University of California, Los Angeles (UCLA)], in whose laboratory some of the work was done with the support of NIH Grant NS043196. We also thank Dr. Paul Burgoyne (MRC National Institute for Medical Research, London, UK) for the Sry transgenic mice; Dr. Andy Greenfield (MRC Mammalian Genetics Unit, Oxfordshire, UK) for Utx and Uty cDNA clones; and R. Bijlani (UCLA), Z. Tornabene (UCLA), M. Cheng [University of Washington (UW)], D. K. Ngnyen (UW), N. Krakauer (University of California, Berkeley), and P. Mann (Tufts) for technical and editorial assistance.

Correspondence should be addressed to Jun Xu, Department of Biomedical Sciences, Cummings School of Veterinary Medicine at Tufts University, North Grafton, MA 01536. E-mail: jun.xu@tufts.edu.

DOI:10.1523/JNEUROSCI.5382-07.2008

Copyright $\odot 2008$ Society for Neuroscience $\quad$ 0270-6474/08/284521-07\$15.00/0 expressed X copy, the Y-linked gene should have the same function and be expressed in similar tissues. In the present study, we compared the expression patterns of Utx (ubiquitously transcribed tetratricopeptide repeat gene on X chromosome) and of Uty (ubiquitously transcribed tetratricopeptide repeat gene on Y chromosome) in adult mouse brain and in cell culture. In addition, we used a mouse model which allows for the separation of sex chromosome-linked gene effects from hormonal effects (Lovell-Badge and Robertson, 1990). In one set of mice, the Y chromosome is deleted for the testis determining gene $S r y$ (thereafter termed $\mathrm{Y}^{-}$), which results in $\mathrm{XY}^{-}$ female mice with ovaries similar to XX females. In a second set of mice, an Sry transgene is inserted into an autosome, resulting in XXSry males with testes similar to XY males.

The UTX protein contains a Jumonji-C domain, a structural motif shared by many histone demethylases (Klose and Zhang, 2007). UTX catalyzes the removal of methylation at lysine 27 of histone $\mathrm{H} 3$, a repressive chromatin modification (Agger et al., 2007; Hong et al., 2007; Lan et al., 2007; Lee et al., 2007); thus, UTX acts as a powerful activator of gene expression. The function of UTY, which has $84 \%$ amino acid sequence similarity with UTX, is unknown.

We found that although expression of Utx and Uty was broadly similar in male mouse brain, there were significant differences in the regional expression patterns. Utx was expressed preferentially in the amygdala, and Uty in the paraventricular nucleus (PVN) of the hypothalamus. These discordances be- 
(a) Utx

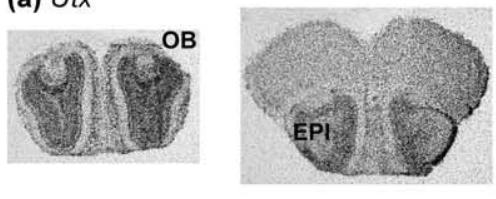

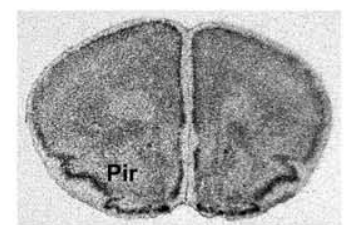
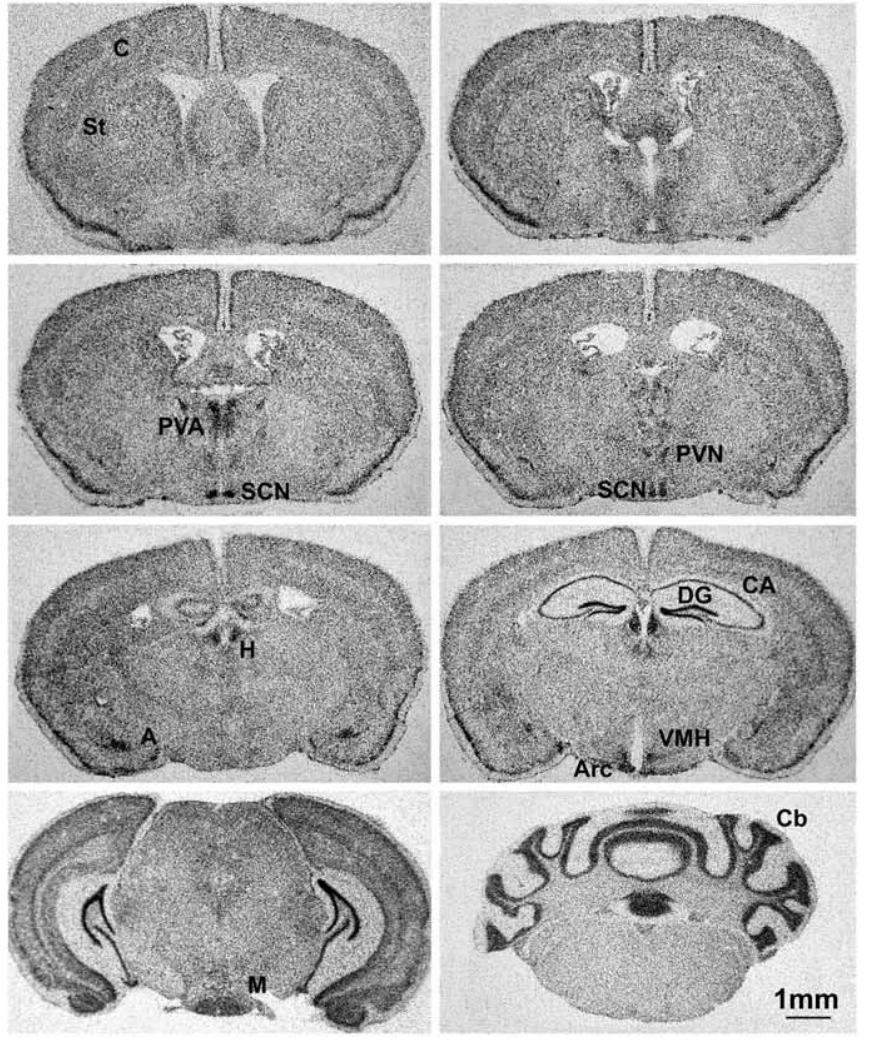
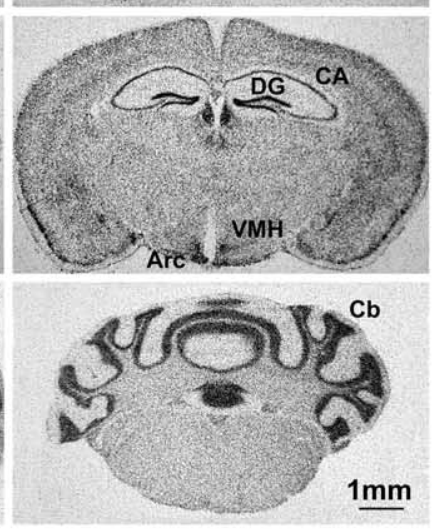

(b) $D d x 3 x$
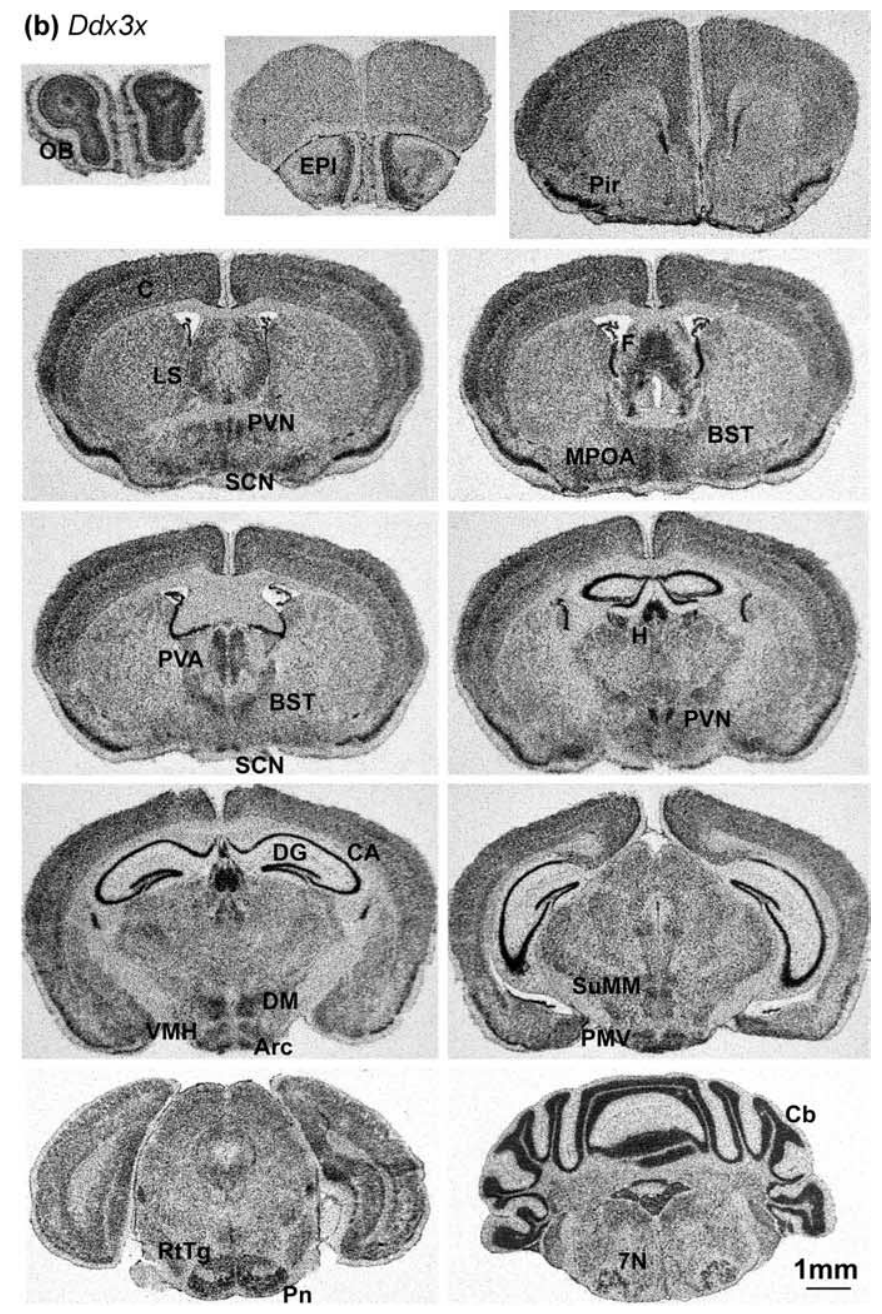

Figure 1. $\quad \boldsymbol{a}, \boldsymbol{b}$, Expression of $U t x(\boldsymbol{a})$ and $D d x 3 x(\boldsymbol{b})$ by in situ hybridization on sections of adult mouse brain. Specific brain regions, including the olfactory bulb (OB), piriform cortex (Pir), habenula (H), hypothalamus [SCN, ventromedial hypothalamic nucleus (VMH), arcuate nucleus (Arc), etc.], hippocampus (CA and DG), and cerebellum (Cb) had a relatively higher expression level. Utx was detected specifically in the amygdala (A). Ddx3x was detected in the fornix (F), whereas Utx was not. 7N, Facial nucleus; BST, bed nucleus of the stria terminalis; C, cortex; DM, dorsomedial hypothalamic nucleus; EPI, external plexiform layer of olfactory bulb; LS, lateral septal nucleus; $M$, mammillary nucleus; MPOA, medial preoptic area; PMV, premammillary nucleus; Pn, pontine nuclei; PVA, paraventricular thalamic nucleus; RtTg, reticulotegmental nucleus; SuMM, supramammillary nucleus; St, striatum.

tween $\mathrm{X}$ and $\mathrm{Y}$ paralogues in specific brain areas may lead to sex differences in brain function.

Discordant brain expression patterns between paralogous genes could arise because of differences in chromatin remodeling on the $\mathrm{X}$ and $\mathrm{Y}$ chromosomes. Both histone methylation and acetylation have been shown to activate or repress expression of certain genes in specific brain regions (Tsankova et al., 2007). Using P19 cells, an in vitro model of neuronal differentiation (McBurney, 1993), we demonstrated that the divergence in the expression between $U t x$ and $U t y$ was associated with differences in the levels of two histone modifications, $\mathrm{H} 3$ dimethylation at lysine 4 (H3K4me2) and H4 acetylation at lysine 16 (H4K16ac).

\section{Materials and Methods}

Animals. Procedures for mouse use were approved by the University of California, Los Angeles, Chancellor's Animal Research Committee. Mice were bred from stocks obtained from Jackson Laboratories (Bar Harbor, ME) (C57BL/6J) or as a gift (MF1 mice) from Dr. Paul Burgoyne [Medical Research Council (MRC), National Institute for Medical Research, London, UK]. Conditions of mouse husbandry, the breeding paradigm to generate sex-reversed and control mice $\left(\mathrm{XY}^{-}\right.$females, $\mathrm{XY}^{-}$Sry males, $\mathrm{XX}$ females, and XXSry males) as well as the modalities of tissue collection are described by Xu et al. (2006). Adult tissues were collected from 8 - to 10-month-old mice, except for the four-core mouse brains, which were from 12- to 14-month-old animals.

Cell culture and neuronal induction. P19 embryonal carcinoma (EC) cells were cultured in DMEM medium containing 10\% fetal bovine serum. Neural differentiation was initiated by plating P19 cells in medium containing $0.3 \mu \mathrm{M}$ retinoic acid (RA; Sigma, St. Louis, MO) on nonadhesive Petri dishes to promote the formation of aggregates. After a $4 \mathrm{~d}$ exposure to RA, the aggregates were dispersed with trypsin (Invitrogen, Carlsbad, CA) and replated on cell culture dishes. Cytosine arabinoside (Ara-C; Sigma), which inhibits the proliferation of non-neuronal cells, was then added to the medium for neuronal selection. P19 neurons appeared to be fully differentiated by day 6 . Cells were collected on day 10 for mRNA and histone modification analyses. Three independent samples were cultured and tested for each cell type.

In situ hybridization. In situ hybridization of brain sections was performed as described by Xu et al. (2006). The riboprobes were transcribed from linearized plasmids containing either a Utx or Uty cDNA insert. Both cDNA clones, originally generated by Dr. A. Greenfield (MRC Mammalian Genetics Unit, Oxfordshire, UK) (Greenfield et al., 1998), were rederived into pT7T3-Pac vectors. The Utx riboprobe corresponds to nucleotides 1284-2205 of GenBank sequence NM_009483 and the Uty riboprobe to nucleotides 1-2392 of sequence NM_009484. Concerned whether the differences in hybridization pattern between the two probes might be related to differences in probe length and location of the 
Table 1. Utx expression in male and female brain measured by in situ hybridization

\begin{tabular}{lll}
\hline & Ratio (female/male) $^{a}$ & Paired $t$ test \\
\hline Cortex & $1.17 \pm 0.14$ & $t_{(7)}=23.8, p<0.0001$ \\
Striatum & $1.23 \pm 0.19$ & $t_{(7)}=18.0, p<0.0001$ \\
SCN & $1.35 \pm 0.14$ & $t_{(7)}=7.3, p<0.001$ \\
Habenula & $1.33 \pm 0.11$ & $t_{(7)}=9.7, p<0.0001$ \\
CA & $1.21 \pm 0.08$ & $t_{(7)}=9.9, p<0.0001$ \\
Dentate gyrus & $1.15 \pm 0.07$ & $t_{(7)}=6.5, p<0.001$ \\
PVN & $1.29 \pm 0.23$ & $t_{(5)}=2.7, p<0.05$ \\
Amygdala & $1.09 \pm 0.08$ & $t_{(6)}=1.1, p=0.30$ \\
\hline
\end{tabular}

${ }^{a}$ Data are mean \pm SEM.

probes in the genes, we designed a shorter Uty probe (1300 bp) by linearizing the plasmid with BsrGI. This Uty probe was close in length to the $U t x$ probe $(922 \mathrm{bp}$ ) and located in a similar region of the gene. No differences in the level or regional distribution of hybridization signals were observed using either of the two Uty probes, suggesting that the differences we detected between Utx and Uty were independent of probe length or target gene region. To ensure specificity, the Uty riboprobe was applied to slides containing adjacent male and female brain sections. Only male sections hybridized, demonstrating specificity of the probe. Expression of $D d x 3 x$, another $\mathrm{X}$-linked escape gene, was also examined on brain sections. The $D d x 3 x$ riboprobe was transcribed from a linearized cDNA clone which contained a $624 \mathrm{bp}$ PCR product (nucleotides 443-1067, GenBank sequence NM_010028) constructed with the pCRScript kit (Stratagene, La Jolla, CA).

Eight brain regions were selected for analysis including the striatum, cortex, suprachiasmatic nucleus (SCN), PVN, habenula, CA1 and CA3 subfields of the hippocampus (CA), dentate gyrus (DG), and amygdala. Cortex expression was quantified on the sections that contained striatum. Each region was compared between eight pairs of males and females except for the amygdala and PVN, for which only seven (amygdala) or six (PVN) pairs were compared. Utx and Uty expression was compared on adjacent sections of six male brains containing the amygdala, PVN, and habenula. The hybridization intensity of the Utx or Uty probe in the amygdala or PVN was averaged and normalized against that in the habenula (which was easily identifiable on the same brain sections). Paired $t$ tests were applied to compare the relative expression of Utx versus Uty.

Northern blots. Northern blot hybridization was done as described previously (Xu et al., 2002). The template for synthesis of the Utx probe was the same 922 bp cDNA fragment used for in situ hybridization. To synthesize the control Gapdh probe, a 451 bp reverse transcriptase (RT)PCR product (nucleotides 520-971, GenBank sequence XM_111622) was used as template. Gapdh is ubiquitously expressed across tissues and at different ages and there is no detectable sex difference in its expression (Xu et al., 2002). Quantification of band intensity was done as described previously (Xu et al., 2002). For comparison of gene expression in adult liver between wild-type males and females, four samples were tested in each sex and each sample was pooled from two mice. The neonatal brain blot contained four mRNA samples for each sex and each sample was derived from three to four day 1 pups. On the Northern blot of four core mouse brains, each genotype was represented by three samples, each sample being pooled from two adult mice.

Quantitative RT-PCR and expression arrays. Total RNA was isolated with an RNeasy kit (Qiagen, Valencia, CA) and was then reversetranscribed using a first-strand synthesis kit (Invitrogen). The amount of Utx and Uty mRNA in P19 stem and neuron samples was determined on a LightCycler system (Roche, Indianapolis, IN). The forward and reverse primer sequences, obtained from the Primer Bank website (http:// pga.mgh.harvard.edu/primerbank/), for Utx were $5^{\prime}$-AAG GCT GTT CGC TGC TAC G-3' and 5'-GGA TCG ACA TAA AGC ACC TCC-3'; for Uty, $5^{\prime}$-GTT TTG TGG CAT GGG AGG ATA-3' and 5'-GAT GGC ACT GTC TCA GGT GG-3'; and for Gapdh, $5^{\prime}$-AGA GAG AGG CCC

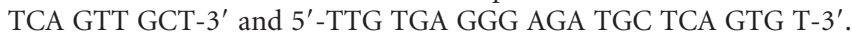
Efficiencies of primer pairs judged by serial dilutions were similar. The level of Gapdh mRNA serves as a reference for sample loading. PCRs were repeated at least two times independently. Expression was compared between Utx and Uty using a paired $t$ test in six P19 samples (three undifferentiated and three P19 neuron samples). Expression array data on the undifferentiated P19 cells and P19 neurons were obtained using two Agilent (Palo Alto, CA) mouse gene expression 44K arrays and two Affymetrix (Santa Clara, CA) GeneChip Mouse Genome 4302.0 arrays following standard protocols. Analysis of arrays was done at the Microarray Center at the University of Washington.

ChIP assays. Chromatin was extracted from three P19 stem cell samples and three P19 neuron samples following the manufacturer's instruction (EZ-ChIP kit; Millipore, Billerica, MA). Briefly, $20 \mu \mathrm{g}$ of fixed chromatin was incubated for $15 \mathrm{~h}$ at $4^{\circ} \mathrm{C}$ with $5 \mu \mathrm{g}$ of antibody (antidimethylated $\mathrm{H} 3$ at lysine 4 and anti-acetylated $\mathrm{H} 4$ at lysine 16, Millipore). After a second incubation with protein A-Sepharose beads (GE Healthcare, Little Chalfont, Buckinghamshire, UK) for $2 \mathrm{~h}$ and three rinses in wash buffer, bound DNA was eluted from the protein A beads by agitation in $1 \%$ SDS for $15 \mathrm{~min}$. Chromatin immunoprecipitation (ChIP) DNA was purified, and then used as template in real-time PCR quantification of histone modifications using a Roche LightCycler. Utxand $U t y$-specific PCR primers were designed to amplify regions spanning from nucleotides -328 through -148 on the Utx promoter and from nucleotides - 364 through -231 on the Uty promoter. The sequences of the primers used are as follows: $5^{\prime}$-CTC CAT CTC TGC CTG TGT ATT T-3' (Utx forward); 5'-CCA CTT AAC TTG CTA GCT GCT TT-3' (Utx reverse); 5' -TTA GTG CTC TTT GTT CCT TGT GAG-3' (Uty forward); and 5'-CTA AGA GCT CTA CCT ACC GAC CAC-3' (Uty reverse). Efficiencies of primer pairs judged by serial dilutions were similar. The enrichment of $\mathrm{H} 3 \mathrm{~K} 4 \mathrm{me} 2$ and $\mathrm{H} 4 \mathrm{~K} 16 \mathrm{ac}$ was normalized against the input, which served as an internal control for possible differences in amplification efficiency between primer pairs. Histone modifications were compared between Utx and Uty using a paired $t$ test in six P19 samples (three stem cell samples and three neuron samples). DNA from chromatin fractions obtained for histone $\mathrm{H} 4$ acetylation at lysine 16 was also submitted to Nimblegen (Madison, WI) for array analysis using the manufacturer conditions. Analysis was done using the manufacturer software.

\section{Results}

\section{Expression of $U t x$ and $D d x 3 x$ in mouse brain}

Using in situ hybridization with specific antisense riboprobes, the expression patterns of $U t x$ and $D d x 3 x$ were examined in adult mouse brain. In human, both UTX and DDX3X have been proposed as candidates for the facial emotion recognition deficiency found in Turner syndrome women (Good et al., 2003). These women have only one X chromosome and would therefore express $\mathrm{X}$ escapee genes at a reduced level relative to XX females. Although Utx and $D d x 3 x$ were found to be expressed throughout the brain, higher expression levels were apparent in specific regions (Fig. 1), including the olfactory bulb, piriform cortex, habenula, hypothalamus [SCN, ventromedial hypothalamic nucleus, arcuate nucleus, etc], hippocampus (DG and CA), and cerebellum. This is, in part, because of a higher cell density in areas such as hippocampus and cerebellum. Utx and $D d x 3 x$, showed distinct expression patterns; for instance, $D d x 3 x$, but not $U t x$, was expressed in the fornix (Fig. $1 a, b$ ).

The specificity of the $U t x$ and $D d x 3 x$ riboprobes was verified by Northern blots on which specific hybridization bands were detected, distinct from those of their Y-linked paralogues Uty and $D d x 3 y$ (Xu et al., 2002). Furthermore, no hybridization signal was detected on brain sections when sense-strand riboprobes were used (data not shown).

\section{Sex-specific differences in Utx expression in the cortex, striatum, SCN, habenula and hippocampus, but not in the amygdala}

The amygdala-specific expression of Utx prompted a detailed analysis of its expression, which was quantified on in situ hybridization autoradiograms in individual brain regions including the 
cortex, striatum, SCN, habenula, CA, and DG, as well as the amygdala. Females expressed Utx at a significantly higher level (ranging from 13 to $35 \%$ higher) than males in almost all of the regions tested (Table 1, Fig. 2). However, no significant sex-specific difference was found in the amygdala (Table 1, Fig. 2). Note that the expression levels shown in Figure 2 should not be directly compared across different brain regions, because the background hybridization intensity was measured in different brain areas and then subtracted from that of the region being quantified.

We have found previously, using Northern blot analyses, that Utx is expressed at a higher level in the brain of adult females compared with males (Xu et al., 2002). The female bias for Utx expression was confirmed in the present study both, in neonatal brain as well as in adult liver (Fig. 3) (neonatal brain, mean \pm SEM]: $5.7 \pm 0.6$ (male) vs $8.2 \pm 0.3$ (female), $t_{(6)}=4.0, p<0.01$; adult liver: $3.6 \pm 0.3$ (male) vs $5.6 \pm 0.7$ (female), $t_{(6)}=3.0, p<0.05$ ), indicating that the sex-specific difference in Utx expression exists throughout development and in different tissues.

\section{Sex-specific differences in Utx expression depend on the complement of sex chromosomes}

To confirm that the higher expression of Utx in females is indeed caused by the presence of two expressed copies of the gene, we compared Utx expression in gonadally intact adult mice between 12 and 14 months of age using the four core genotypes (i.e., XX females, $\mathrm{XY}^{-}$females, XXSry males, and $\mathrm{XY}^{-}$Sry males). Three independent samples, each from two adult brains, were tested by Northern blotting for each genotype. A two-way ANOVA revealed a main effect of the sex chromosome complement, i.e., XX vs XY, on Utx expression relative to Gapdh (Fig. 4$)\left(F_{(1,8)}=7.04\right.$; $p<0.05)$. Mice with two X chromosomes had higher levels of Utx mRNA than those with one X chromosome. There was no effect of gonadal sex (male vs female). However, expression in mice with two $\mathrm{X}$ chromosomes was not doubled, indicating that expression from the inactive X chromosome was significantly lower (15-35\%) than that from the active $\mathrm{X}$ chromosome (Fig. 2, Table 1).

\section{Differential expression of Utx and Uty in the amygdala and PVN in male mouse brain}

We compared the expression pattern of Uty to that of Utx on adjacent sections from adult male mouse brains using in situ hybridization. In general, the expression patterns were similar for the two paralogues (Fig. 5), suggesting that Uty may compensate for the increased expression of Utx in females. In particular, Uty was well expressed in the hippocampus, habenula, hypothalamic nuclei, and cerebellum. However, there were notable differences between the paralogues: Uty was expressed more highly in the PVN, but less in the amygdala, relative to Utx (Fig. 5). Expression levels were quantified in six male brains by measuring the hybridization intensity of the riboprobes in the amygdala, PVN, and habenula on sections that contained all three regions. Using the habenula as a reference area, $U t x$ was expressed at a significantly higher level in the amygdala than Uty (Table 2) $\left(t_{(5)}=9.10 ; p<\right.$ $0.001)$, whereas in the PVN, Uty was expressed more highly than $U t x$ (Table 2) $\left(t_{(5)}=-5.10 ; p<0.01\right)$.
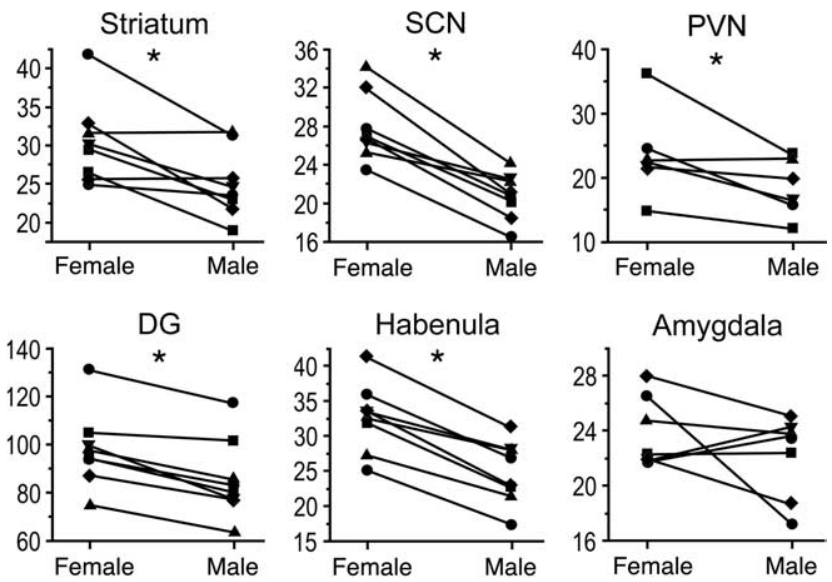

Figure 2. Utx expression quantified by in situ hybridization to sections of male and female adult mouse brains. Female mice

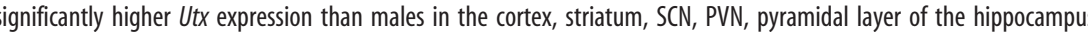
(CA1/CA3), DG, and habenula, but not in the amygdala. The in situ hybridization labeling intensity was measured as the mean pixel density minus background in six to eight pairs of females and males processed in parallel on the same slides. ${ }^{*} p<0.05$.

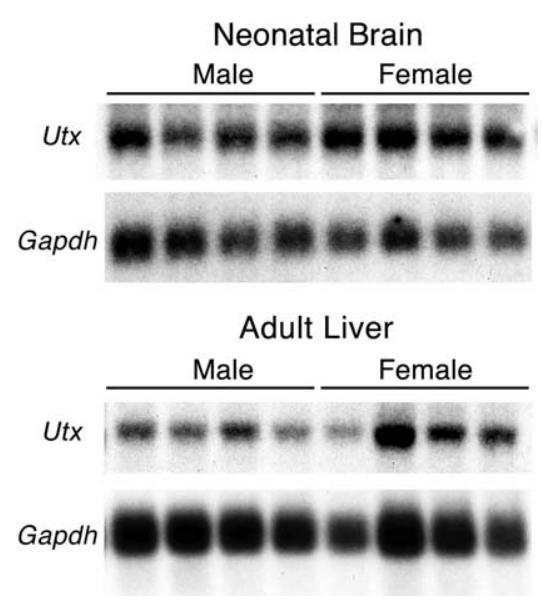

Figure 3. Utx expression in male and female neonatal mouse brains and in adult livers. Expression determined by Northern blot analysis was higher in females compared with males. Gapdh was used as a loading control.
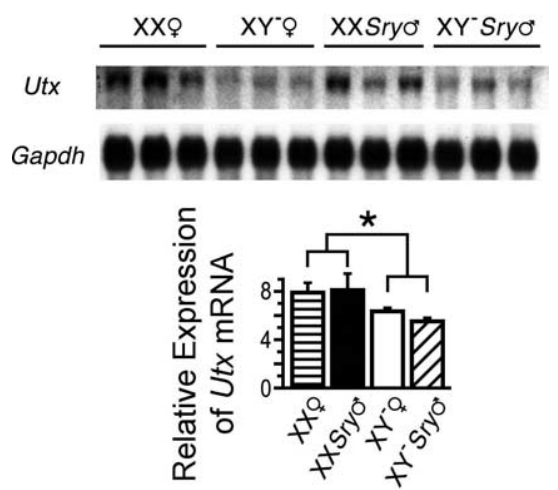

Figure 4. Utx expression in brains from XX females, $X Y^{-}$females, XXSry males, and $X Y^{-}$Sry males. Each of the three samples per genotype was derived from two adult brains. Gapdh was used as a loading control. Error bars indicate SEM. ${ }^{*} p<0.05$.

Expression and histone modifications of Utx and Uty in the neural differentiation of P19 EC cells

P19 EC cells are pluripotent cells with a normal 40,XY male karyotype, which can be readily induced to differentiate into neurons. P19 cells were analyzed to determine whether the differ- 

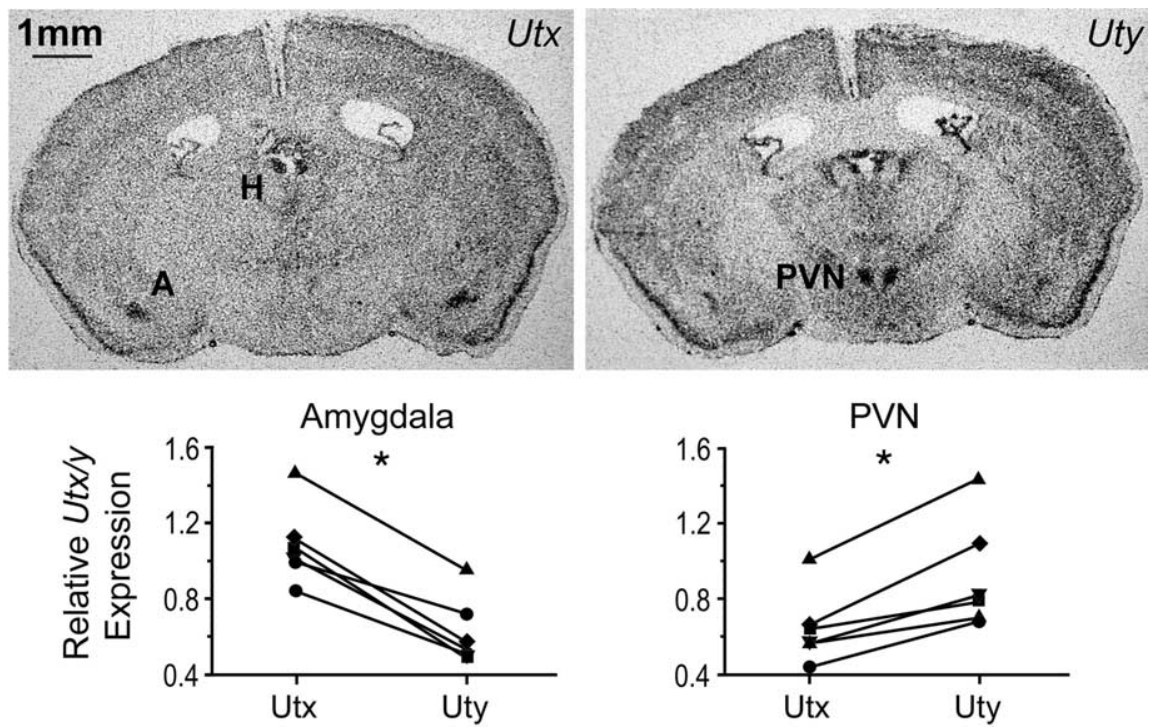

Figure 5. Utx and Uty differential expression in male mouse brain. Top, Expression patterns of Uty and Utx by in situ hybridization on adjacent adult male brain sections. Uty was expressed more highly in the PVN, but less in the amygdala (A) relative to $U t x$. The graphs below show the quantification of expression levels of Uty and Utx in the amygdala and PVN, using the habenula $(H)$ as a reference area, based on sections that contained all three regions $(n=6) .{ }^{*} p<0.05$.

Table 2. Expression of Utx and Uty in the amygdala and PVN, relative to the habenula, in adult male mice measured by in situ hybridization

\begin{tabular}{llll}
\hline & $\begin{array}{l}\text { Utx } \\
\text { (mean } \pm \text { SEM) }\end{array}$ & $\begin{array}{l}\text { Uty } \\
\text { (mean } \pm \text { SEM) }\end{array}$ & Paired $t$ test \\
\hline Amygdala & $1.08 \pm 0.09$ & $0.63 \pm 0.18$ & $t_{(5)}=9.1, p<0.0005$ \\
PVN & $0.64 \pm 0.08$ & $0.82 \pm 0.12$ & $t_{(5)}=5.1, p<0.0005$ \\
\hline
\end{tabular}

ences in brain expression patterns between Utx and Uty described above were associated with distinct epigenetic modifications of the paralogues. Using real-time quantitative PCR, we determined that Utx mRNA was much more abundant than Uty mRNA both in undifferentiated P19 cells and in P19 neurons (Utx, mean \pm SEM, $292 \pm 78$; Uty, $0.08 \pm 0.03$; paired $t$ test, $t_{(5)}=3.7 ; p=$ $0.01)$. Expression of $U t x$ and $U t y$ in P19 cells estimated on Agilent mouse 44K expression arrays and on Affymetrix mouse 4302.0 GeneChips was consistent with the quantitative RT-PCR results, i.e., Utx was expressed more highly than Uty both in undifferentiated P19 cells (Agilent, Utx/Uty $=7.3 \pm 0.9$ and Affymetrix, $U t x / U t y=13.5$ ) and in neurons (Agilent, $U t x / U t y=5.7 \pm 0.1$; Affymetrix, Utx/Uty = 10.9), although the difference between the paralogues was lower than when measured by real-time PCR. No significant difference in expression of Utx or Uty was detected between P19 neurons and P19 stem cells, either by RT-PCR (Utx, $\left.t_{(4)}=2.46, p=0.06 ; U t y, t_{(4)}=1.54, p=0.20\right)$, or by analysis of array data.

To determine whether the higher expression of Utx versus Uty was associated with chromatin differences, we examined two histone marks characteristic of transcription activation, H3K4me2 and $\mathrm{H} 4 \mathrm{~K} 16 \mathrm{ac}$, at the promoter regions of Utx and Uty using quantitative PCR analysis of ChIP fractions. For the six ChIP samples, three stem cell samples, and three P19 neuron samples, a pair $t$ test between the paralogues indicated a trend of higher level of $\mathrm{H} 3 \mathrm{~K} 4 \mathrm{me} 2$ on the $U t x$ promoter relative to $U t y\left(t_{(5)}=2.4\right.$; $p=0.06)$. H3K 4 me 2 was also found to be higher at the Utx promoter, but not the Uty promoter, in neurons versus stem cells (P19 stem, $0.42 \pm 0.08$; neuron, $1.27 \pm 0.17 ; t_{(4)}=4.4 ; p=0.01$ in the case of $U t x)$. There were no apparent differences in $\mathrm{H} 4 \mathrm{~K} 16 \mathrm{ac}$ at the 5 ' end of the two genes. However, a ChIP-on-chip analysis on DNA tiling arrays, which represent the whole mouse $\mathrm{X}$ and $\mathrm{Y}$ chromosomes, showed a significantly higher accumulation of H4K16ac along the Utx gene body compared with Uty, the latter showing signals no higher than background (data not shown).

\section{Discussion}

Utx is one of a handful of X-linked genes that escape $\mathrm{X}$-inactivation in both humans and mice (Disteche et al., 2002; Brown and Greally, 2003). In this study, we determined that Utx was expressed at a higher level in female than male mice in specific brain regions such as the cortex, hippocampus, and hypothalamus. A sexspecific difference in expression of Utx was also found in developing brains as well as in adult livers. The sex chromosome complement, not the gonadal sex, dictated the expression of Utx as demonstrated by higher expression in XX than XY mice, regardless of their gonadal type. We cannot, however, exclude the possibility of a gonadal steroid effect on Utx expression because we have not specifically tested the effect of steroids by manipulating their levels in gonadectomized mice, and did not record the estrous state of the females. Although the most likely explanation for the XX versus $\mathrm{XY}$ difference in expression is the difference in the genomic dose of Utx, it is also possible that a Y-linked factor, such as UTY, suppresses the expression of Utx in XY mice. The sex-specific differences in expression of Utx may not necessarily lead to differences at the protein level: we have shown previously that Eif2s $3 x$, another X escapee gene, is more highly expressed in females than in males for mRNA only but not for the EIF2S3X protein (Xu et al., 2006). In addition, the effects of sex-linked genes and sex hormones may cancel each other out to yield similar physiology between the sexes. For instance, immune responses and cytokine production induced by autoantigens are enhanced in mice with an XY complement compared with mice with an XX complement, but such immune reactions are repressed by male sex hormones (Palaszynski et al., 2005).

The sex-specific differences in expression of an $\mathrm{X}$ escapee could conceivably be compensated for by the expression of a functionally similar Y-linked homolog in males (Arnold et al., 2004; Xu et al., 2005). However, biochemical analyses of UTX and UTY have shown that, whereas UTX demethylates H3K27, UTY does not appear to have this function (Hong et al., 2007; Lan et al., 2007). Our studies also show differences between Utx and Uty in terms of their expression patterns in brain. Although we found that Utx and Uty are expressed in a parallel manner for many brain regions, we also found brain regions where Utx and Uty had distinct expression patterns. In the amygdala, Utx had a higher expression than Uty, whereas in the PVN, Uty was expressed at a significantly higher level than $U t x$. The male-specific high expression of Uty in the PVN represents the first example of a Y-linked gene expressed in a male-typical manner and may play a role in sexual differentiation of the brain. The discordance in transcription between the $\mathrm{X}$ and $\mathrm{Y}$ paralogues suggests that they are not coregulated, which may be because of differences in gene struc- 
ture (promoter, enhancer, $3^{\prime}$ end), epigenetic modifications, and/or tissue-specific transcriptional factors.

As a proof of principle, we tested two histone marks associated with active chromatin, $\mathrm{H} 3 \mathrm{~K} 4 \mathrm{me} 2$ and $\mathrm{H} 4 \mathrm{~K} 16 \mathrm{ac}$, at the Utx and Uty genes in P19 stem cells and neurons. In both cell types, $\mathrm{H} 3 \mathrm{~K} 4 \mathrm{me} 2$ exhibited a trend of higher enrichment at the 5 ' end of Utx compared with Uty, and H4K16ac was enriched over the Utx gene body, but not over Uty. This is consistent with the higher expression of Utx relative to Uty. Because P19 cells are derived from a day 7 mouse embryo (McBurney, 1993), the expression and epigenetic modifications of Utx and Uty seem to become divergent at very early stages of development. It is not clear whether similar epigenetic differences between the paralogues will also be found in specific brain regions in adult mice. ChIP assays in discrete brain regions will be needed to determine whether epigenetic modifications vary in a regional manner in vivo.

Interestingly, we detected Utx expression in the amygdala where other X-linked escapee genes, such as Eif2s $3 x$, are not expressed (Xu et al., 2006). Studies have shown that the amygdala plays an important role in human emotion and social behavior, including recognition of other people's emotions from facial expressions (Adolphs, 2003). The amygdala is activated in functional brain imaging when photos of fearful faces are presented to subjects (Morris et al., 1996). When the amygdala is damaged bilaterally, one loses the ability to recognize emotions from other people's facial expressions (Anderson and Phelps, 2000). The molecular mechanism responsible for the role of the amygdala in social cognition is not clear. The $\mathrm{X}$ chromosome might play a role in normal development of the amygdala and, in turn, emotional facial recognition.

45,X Turner Syndrome women have been recognized for some time to have varying degrees of impairment in their ability to recognize emotional facial expressions (Ross et al., 2000), which was later correlated with a significant increase in amygdala volume (Good et al., 2003; Kesler et al., 2004). It is thus proposed that certain X escapee genes might influence the development of the amygdala, and therefore Turner women who are haploinsufficient for these $\mathrm{X}$ escapees are affected in their social cognitive ability (Skuse et al., 2003). By examining a group of Turner women with partial deletions on the X chromosome, Good et al. (2003) identified a $4.96 \mathrm{Mb}$ critical interval that is specifically important for amygdala development and social cognition. Among 21 confirmed genes within this interval, EFHC2 is a leading candidate for the social cognition phenotype (Weiss et al., 2007). Other candidates are USP9X, DDX3X, and UTX, which escape $\mathrm{X}$ inactivation in human. USP9X is a deubiquitinating enzyme known for its involvement in synaptic development. However, in a previous study we did not detect USP9X protein in mouse amygdala (Xu et al., 2005). In the present study, we examined the expression pattern of two other genes in the critical interval, $D d x 3 x$ and Utx. Utx was expressed in the amygdala at an impressive level, whereas $D d x 3 x$ was detected at a lower level. Thus, provided that its expression pattern is similar in human and mouse, Utx is an attractive candidate for the abnormalities in amygdala in Turner syndrome.

Social cognition, aggression, and parental behavior are among an increasing list of sexually dimorphic traits, the origin of which is not always clear (Skuse et al., 1997; Gatewood et al., 2006). Female mice with an XY karyotype are faster to attack an intruder in an aggression test and are slower to retrieve pups in a maternal task when compared with XX female mice (Gatewood et al., 2006). Because the amygdala is intrinsically involved in both ag- gressive and parental behavior (Byrnes et al., 2007; Nelson and Trainor, 2007), the specific expression of Utx we found suggests that this gene may play a role in these behavioral sexual dimorphisms.

The majority of sex-specific differences in the brain have been thought and in many cases proven to be mediated by the actions of steroid hormones (Arnold and Gorski, 1984; Morris et al., 2004). However, as shown in the present study, genes on the $X$ and $\mathrm{Y}$ chromosomes may be an additional source of sex-specific signals (Arnold, 2004). The possibility of sexual dimorphisms induced by sex-linked genes is particularly relevant to brain function, because of the relatively high concentration of brain genes on the X chromosome (threefold higher than on autosomes) (Zechner et al., 2001) and the higher expression of X-linked genes in brain (Nguyen and Disteche, 2006). Trimethylation at histone $\mathrm{H} 3 \mathrm{~K} 27$, which is removed by UTX, is associated with repressed chromatin, in particular the inactive X chromosome (Heard and Disteche, 2006). Removal of this repressive mark would be important specifically in females to allow for X chromosome reactivation and for escape from $\mathrm{X}$ inactivation. This could explain why Utx itself escapes from $\mathrm{X}$ inactivation, leading to its higher expression in females compared with males. The specific role(s) of $U t x / U t y$ in brain will need to be further studied. The retention of a Y-linked paralogue together with escape from $\mathrm{X}$ inactivation of the X paralogue may afford greater flexibility in gene expression level and possibly functional difference between the sexes.

\section{References}

Adolphs R (2003) Cognitive neuroscience of human social behaviour. Nat Rev Neurosci 3:165-178.

Agger K, Cloos PA, Christensen J, Pasini D, Rose S, Rappsilber J, Issaeva I, Canaani E, Salcini AE, Helin K (2007) UTX and JMJD3 are histone $\mathrm{H} 3 \mathrm{~K} 27$ demethylases involved in HOX gene regulation and development. Nature 449:731-734.

Anderson AK, Phelps EA (2000) Expression without recognition: contributions of the human amygdala to emotional communication. Psychol Sci 11:106-111.

Arnold AP (2004) Sex chromosomes and brain gender. Nat Rev Neurosci 5:701-708.

Arnold AP, Gorski RA (1984) Gonadal steroid induction of structural sex differences in the central nervous system. Annu Rev Neurosci 7:413-442.

Arnold AP, Xu J, Grisham W, Chen X, Kim YH, Itoh Y (2004) Sex chromosomes and brain sexual differentiation. Endocrinology 145:1057-1062.

Becker JB, Arnold AP, Berkley KJ, Blaustein JD, Eckel LA, Hampson E, Herman JP, Marts S, Sadee W, Steiner M, Taylor J, Young E (2005) Strategies and methods for research on sex differences in brain and behavior. Endocrinology 146:1650-1673.

Brown CJ, Greally JM (2003) A stain upon the silence: genes escaping X inactivation. Trends Genet 19:432-438.

Byrnes EM, Lee JO, Bridges RS (2007) Alterations in $\mathrm{GABA}_{\mathrm{A}}$ receptor alpha2 subunit mRNA expression following reproductive experience in rats. Neuroendocrinology 85:148-156.

Carrel L, Willard HF (2005) X-inactivation profile reveals extensive variability in X-linked gene expression in females. Nature 434:400-404.

Disteche CM, Filippova GN, Tsuchiya KD (2002) Escape from X inactivation. Cytogenet Genome Res 99:36-43.

Gatewood JD, Wills A, Shetty S, Xu J, Arnold AP, Burgoyne PS, Rissman EF (2006) Sex chromosomes complement and gonadal sex influence aggressive and parental behaviors in mice. J Neurosci 26:2335-2342.

Good CD, Lawrence K, Thomas NS, Price CJ, Ashburner J, Friston KJ, Frackowiak RS, Oreland L, Skuse DH (2003) Dosage-sensitive X-linked locus influences the development of amygdale and orbitofrontal cortex, and fear recognition in humans. Brain 126:2431-2446.

Greenfield A, Carrel L, Pennisi D, Philippe C, Quaderi N, Siggers P, Steiner K, Tam PP, Monaco AP, Willard HF, Koopman P (1998) The UTX gene escapes X inactivation in mice and humans. Hum Mol Genet 7:737-742.

Heard E, Disteche CM (2006) Dosage compensation in mammals: finetuning the expression of the X chromosome. Genes Dev 20:1848-1867. 
Hong S, Cho YW, Yu LR, Yu H, Veenstra TD, Ge K (2007) Identification of JmjC domain-containing UTX and JMJD3 as histone H3 lysine 27 demethylases. Proc Natl Acad Sci USA 104:18439-18444.

Kesler SR, Garrett A, Bender B, Yankowitz J, Zeng SM, Reiss AL (2004) Amygdala and hippocampal volumes in Turner syndrome: a highresolution MRI study of X-monosomy. Neuropsychologia 42:1971-1978.

Klose RJ, Zhang Y (2007) Regulation of histone methylation by demethylimination and demethylation. Nat Rev Mol Cell Biol 8:307-318.

Lan F, Bayliss PE, Rinn JL, Whetstine JR, Wang JK, Chen S, Iwase S, Alpatov R, Issaeva I, Canaani E, Roberts TM, Chang HY, Shi Y (2007) A histone $\mathrm{H} 3$ lysine 27 demethylase regulates animal posterior development. Nature 449:689-694.

Lee MG, Villa R, Trojer P, Norman J, Yan KP, Reinbert D, Di Croce L, Shiekhattar R (2007) Demethylation of H3K27 regulates polycomb recruitment and H2A ubiquitination. Science 318:447-450.

Lovell-Badge R, Robertson E (1990) XY female mice resulting from a heritable mutation in the primary testis-determining gene, Tdy. Development 109:635-646.

Lyon MF (1961) Gene action in the X-chromosome of the mouse (Mus musculus L). Nature 190:372-373.

McBurney MW (1993) p19 embryonal carcinoma cells. Int J Deve Biol 37:135-140.

Morris JA, Jordan CL, Breedlove SM (2004) Sexual differentiation of the vertebrate nervous system. Nat Neurosci 7:1034-1039.

Morris JS, Frith CD, Perrett DI, Rowland D, Young AW, Calder AJ, Dolan RJ (1996) A differential neural response in the human amygdale to fearful and happy facial expressions. Nature 383:812-815.

Nelson RJ, Trainor BC (2007) Neural mechanisms of aggression. Nat Rev Neurosci 8:536-546.

Nguyen DK, Disteche CM (2006) Dosage compensation of the active X chromosome in mammals. Nat Genet 38:47-53.
Palaszynski KM, Smith DL, Kamrava S, Burgoyne PS, Arnold AP, Voskuhl RR (2005) A yin-yang effect between sex chromosome complement and sex hormones on the immune response. Endocrinology 146:3280-3285.

Ross J, Zinn A, McCauley E (2000) Neurodevelopmental and psychosocial aspects of Turner syndrome. Ment Retard Dev Disabil Res Rev 6:135-141.

Skuse D, Morris J, Lawrence K (2003) The amygdale and development of the social brain. Ann NY Acad Sci 1008:91-101.

Skuse DH, James RS, Bishop DV, Coppin B, Dalon P, Aamodt-Leeper G, Bacarese-Hamilton M, Creswell C, McGurk R, Jacobs PA (1997) Evidence from Turner's syndrome of an imprinted X-linked locus affecting cognitive function. Nature 387:705-708.

Tsankova N, Renthal W, Kumar A, Nestler EJ (2007) Epigenetic regulation in psychiatric disorders. Nat Rev Neurosci 8:355-367.

Weiss LA, Purcell S, Waggoner S, Lawrence K, Spektor D, Daly MJ, Sklar P, Skuse D (2007) Identification of EFHC2 as a quantitative trait locus for fear recognition in Turner syndrome. Hum Mol Genet 16:107-113.

$\mathrm{Xu}$ J, Disteche CM (2006) Sex differences in brain expression of X- and Y-linked genes. Brain Res 1126:50-55.

Xu J, Burgoyne PS, Arnold AP (2002) Sex differences in sex chromosome gene expression in mouse brain. Hum Mol Genet 11:1409-1419.

Xu J, Taya S, Kaibuchi K, Arnold AP (2005) Sexually dimorphic expression of Usp9x is related to sex chromosome complement in adult mouse brain. Eur J Neuro 21:3017-3022.

Xu J, Watkins R, Arnold AP (2006) Sexually dimorphic expression of the $\mathrm{X}$-linked gene Eif2s3x mRNA but not protein in mouse brain. Gene Expr Patterns 6:146-155.

Zechner U, Wilda M, Kehrer-Sawatzki H, Vogel W, Fundele R, Hameister $\mathrm{H}$ (2001) A high density of X-linked genes for general cognitive ability: a run-away process shaping human evolution? Trends Genet 17: 697-701. 